\title{
Hyaluronic Acid versus Alpha-2-Macroglobulin Intra- Articular Injections for Amelioration of Knee Posttraumatic Osteoarthritis: A Rat Model
}

Shaowei Wang ( $\sim$ dreamkobe@163.com)

Shanxi Medical University Second Affiliated Hospital https://orcid.org/0000-0002-1267-9554

Mengbo Zhu

Shanxi Medical University Second Affiliated Hospital

Yanjing Guo

Shanxi Medical University

Ruijia Yang

Shanxi Medical University

Yaqiong Chang

Shanxi Medical University Second Affiliated Hospital

Bin Zhao

Shanxi Medical University Second Affiliated Hospital

Zhenyu Wang

Shanxi Medical University Second Affiliated Hospital

Lei Wei

Brown University

\section{Research article}

Keywords: Osteoarthritis, Alpha-2-macroglobulin, Hyaluronic Acid, PTOA, cartilage degeneration, Rat ACLT OA model

Posted Date: June 25th, 2020

DOl: https://doi.org/10.21203/rs.3.rs-36942/v1

License: (c) (i) This work is licensed under a Creative Commons Attribution 4.0 International License. Read Full License 


\section{Abstract}

Background: The study was performed to evaluate whether intra-articular injection of A2M has better effect than current commonly used Hyaluronic Acid (HA) injection therapy to attenuate cartilage degeneration in a rat anterior cruciate ligament transection (ACLT) osteoarthritis (OA) model.

Method: In vivo effects of $\mathrm{A} 2 \mathrm{M}$ and $\mathrm{HA}$ on cartilage degeneration were evaluated in rat surgery induced ACLT OA models. 100 rats were randomly divided into four groups: (a) Sham surgery + saline (Sham + S), (b) ACLT + A2M, (c) ACLT+HA, or (d) ACLT + saline (ACLT+S). The animals were sacrificed at 12 weeks after surgery. Histological staining was performed to assess cartilage damage. The concentration of MMP-13 and SGAG in synovial fluid lavages was measured using ELISA and spectrophotometric quantitative determination. OA-related gene expression was quantified by qPCR.

Result: Indian ink staining showed that articular cartilage surface treated by A2M was relatively intact compared with the animals treated by ACLT with saline or HA injection. Histological staining indicated that early supplemental intra-articular injection of A2M attenuated OA pathogenesis in the rat ACLT model compared with the animals treated with saline and HA. However, supplemental intra-articular injection of HA showed no significant effect on cartilage protection for post traumatic OA compared with saline treatment. Elisa results showed A2M reduced the concentration of MMP-13 in synovial fluid compared with HA treatment group and other groups. RT-qPCR indicated that supplemental intra-articular A2M inhibits catabolism and enhances anabolic metabolism, while there was no significant difference in the expression of OA-related genes between ACLT+HA group and ACLT+S group.

Conclusion: In rat model, intra-articular injection of A2M had obvious protective effects on cartilage degeneration compared with HA treatment. Major indexes of joint degeneration decreased, providing strong evidence for its intra-articular inhibitory effect. Meanwhile, we found no significant alleviation of articular cartilage pathogenesis in HA treated group, which suggests that the efficacy of HA is questionable and possibly transient, although it is extensively used to improve syndromes.

\section{Background}

Osteoarthritis $(\mathrm{OA})$ is a condition characterized by the progressive destruction of the articular cartilage that lines the knee joints, the subchondral bone surfaces, and synovium [1]. Initially, joints pain is the main symptom of the osteoarthritis. As the disease continue to advance, patients would undergo the reduction in function, might even the loss of ability to complete activities [2, 3]. A wide range of studies have confirmed that joint injury is the critical risk factor for development of OA [4]. Intra-articular inflammation induced by joint injury frequently leads to chronic progressive cartilage degeneration., namely post-traumatic OA. In the acute phase of ( $0-1$ day post injury), relatively high levels of inflammatory mediators, such as IL-1 $\beta \square I L-6 \square I L-8 \square T N F a$, can be observed in synovial fluid (SF) and joint tissues [5]. Those inflammatory mediators maintain a high level for a long term after trauma. The overexpression of SF IL-1, the cardinal inducer of the intra articular inflammation, lasts for three months 
after injury, and the IL-6 and IL-8 lasts for six [6]. Although, levels of protective cytokines also increase after joint injury, this protection mechanism occurs only in early stage of injury. SF IL-10 level drops to normal in two weeks post trauma, and SF level of IL-1 receptor antagonist (IL-1Ra) is below normal after three weeks [7].

Additionally, Matrix Metalloproteinases (MMPS) produced by chondrocyte and synovial cell result in the loss of the normal cartilage tissue, exacerbating the progressive cartilage degeneration. Intensive studies demonstrated that the MMPs levels dramatically increase in injured joint, and this fact has been validated in various animal models [8-12]. Besides the degeneration of cartilage, Monocyte chemoattractant protein 1(MCP-1) contributes to the joint pain in PTOA $[13,14]$. Therefore, the development of PTOA can be considered as the result of persistent inflammation after trauma. Inflammatory mediators leading to the destruction of cartilage and the lack of inflammation control finally result in the syndrome of PTOA [15].

Hence it is assumed that by removing the destructive inflammatory mediators and enzymes we can reduce the chronic inflammation and cartilage degeneration caused by intra-articular injury. A2M is known as a broad-spectrum proteinase inhibitor found in both serum and synovial fluid, which could be used to attenuate cartilage degeneration. Proteinases inducing chronic inflammation can be captured by A2M molecules, and the A2M-proteinase complex will be purged soon from serum. This protective effect of $\mathrm{A} 2 \mathrm{M}$ is benefiting from the special molecular structure, which is capable of blocking almost all kinds of proteinases [16]. Unfortunately, the concentration of A2M in synovial fluid is much lower than that in serum of normal people, as well as OA patients. Thus, endogenous A2M in joint is not enough to diminish intra-joint inflammation $[17,18]$.

One widely used clinical therapy is visco-supplementation, and the most common way of this direction is injecting HA into the joint as the supplementation of the natural joint lubricant [19]. Hyaluronic Acid (HA) received FDA approval in 1997 for treatment of Osteoarthritis (OA) in the United States. In 2009, the American Academy of Orthopaedic Surgeons (AAOS) conducted a meta-analysis regarding HA's treatment and found the evidence for efficacy was considered inconclusive [20,21]. As of early 2014, the AAOS did not find adequate evidence to support listing HA as indicated for treatment of knee OA [22]. However, by using the same thinking, it is possible that injection of A2M instead of HA as the intraarticular supplementation would be helpful to retard the cartilage degeneration in knee OA process, and A2M would make intra-articular injection therapy with more effectivity and biosafety. The objective of this study is to compare the therapeutic effects of $A 2 M$ and HA on OA in rats with knee arthritis.

\section{Materials And Methods}

This study was approved by the Institutional Review Board and the Institutional Animal Care and Use Committee of the Shanxi Medical University.

\section{Animals}


One hundred 10-week-old male wistar rats (180-230 g/each) were obtained from animal center of Shanxi Medical University (Shanxi, China). The study was performed under a protocol approved by the Shanxi Medical University Animal Research Committee (ARC). Seventy-five animals received anterior cruciate ligament transection (ACLT) operations on the right knee joint and divided into 3 groups ( $n=25$ per group): (1) ACLT with intra-articular saline injection (ACLT + Saline), (2) ACLT with intra-articular Hyaluronic Acid injection (ACLT + HA), (3) ACLT with intra-articular A2M injection (ACLT + A2M). The rest twenty-five animals were received sham operation on the right knee joint with intra-articular saline injection (Sham + Saline). A2M (Cat.10602442001, Roche life science, USA) was dissolved in saline to treat rats in a dose of $2 \mathrm{IU} / \mathrm{kg}(20 \mu \mathrm{L})$. [17] Intra-articular injections were performed immediately following and 3 days after ACLT, and then weekly for 6 weeks. All the animals were administrated $20 \mu \mathrm{L}$ liquid in their right knee joint and were euthanized using a standard $\mathrm{CO}_{2}$ chamber 12 weeks after the operation. In each group, 10 animals were used for Indian ink staining for a gross evaluation of cartilage lesion; 15 animals' tibiae were studied histologically, while their femurs were used for real-time polymerase chain reaction (PCR) assessment.

\section{Surgical procedures}

ACLT was surgically performed in the right hind knee joints of the animals as previously described. [23] Once anesthetized, a $1.5 \mathrm{~cm}$ midline incision was made over the anterior knee. The skin was mobilized to expose the patellar tendon. An incision through the joint capsule was made immediately lateral to the tendon. The ACL was cut with a scalpel. Manual laxity testing verified its functional loss. The joint capsule, fascia, and skin were closed in layers. The right hind knee joints of rats in the sham group were sham-operated using the same approach, but without ACLT. Postoperative analgesia using buprenorphine hydrochloride ( $0.05 \mathrm{mg} / \mathrm{kg} \mathrm{SQ})$ was maintained for at least 3 days. Animals were allowed to bear weight on the limbs as tolerated.

\section{Histology}

After the rats were killed with carbon dioxide, gross morphologic lesions on the femur condyle and tibia plateau ( $n=10$ per group) were visualized with India-ink staining [24]. Tibiae in rest set of knee joints ( $n=$ 15 per group) were fixed in 10\% formalin for 72 hours, followed by decalcification in 10\% EDTA solution, while the femurs were used for used for real-time qPCR assessment. Then, tibiae were hemisected in the midsagittal plane, and each half was embedded in a single block of Paraplast X-tra (Sigma-Aldrich, St Louis, MO, USA). Serial $6-\mu \mathrm{m}$-thick sections were cut at intervals of $0 \mu \mathrm{m}, 100 \mu \mathrm{m}$, and $200 \mu \mathrm{m}$ and collected on positively charged glass slides (Superfrost Plus; Fisher Scientific). Safranin-0/fast green was performed and the severity of cartilage damage was assessed using the OARSI Assessment System (OA score $=$ grade $\times$ stage; range, 0 to 24) [25]. Three independent blinded observers scored each section, and the scores for all of the sections were averaged within each joint.

\section{Immunohistochemistry staining}

Immunohistochemistry was carried out using a Histostain-SP kit (Cat. 959943B Invitrogen) to detect the distribution of types II collagen and MMP-13 in cartilage. The sections were digested by $5 \mathrm{mg} / \mathrm{mL}$ 
hyaluronidase in phosphate buffered saline (PBS) (Sigma-Aldrich) for 20 minutes. Nonspecific protein binding was blocked by incubation with a serum blocking solution. Thereafter, the sections were incubated with specific antibodies against rat types II collagen (Santa Cruz Biotechnology) and MMP-13 (Santa Cruz Biotechnology) respectively, at $4{ }^{\circ} \mathrm{C}$ overnight. The negative control sections were incubated with isotype-matched control serum ( $2 \mu \mathrm{g} / \mathrm{mL}$ ) (R\&D Systems, Minneapolis, MN, USA) in PBS.

Subsequently, the sections were treated sequentially with biotinylated secondary antibody and SP conjugate and then developed in DAB chromogen. Photomicrographs were taken with a Nikon E800 microscope (Nikon, Melville, NY, USA).

\section{Rat articular cartilage Real-time qPCR}

The cartilage samples from rats' femur condyle were ground with a mortar and pestle while liquid nitrogen was supplied. Total RNA was isolated from rat cartilage tissues using an RNeasy isolation kit (Qiagen, Valencia, CA) according to manufacturer's instruction. Cartilage samples from 5 rats were dissected using a scalpel and pooled together, thus there were 3 pooled samples per group. Total RNA was reverse transcribed into first-strand complementary CDNA using the iScript ${ }^{\mathrm{TM}} \mathrm{cDNA}$ synthesis kit (BioRad, Hercules, CA, USA). RT-PCR was performed on a 96-well plate ABI Prism 7500 (Applied Biosystems, Foster City, CA) using SuperReal PreMix reagent (Qiagen, Valencia, CA) according to the manufacturer's protocols. The total volume $(20 \mu \mathrm{L})$ of each PCR reaction contained $10 \mu \mathrm{L}$ SuperReal PreMix, $7 \mu \mathrm{L} \mathrm{ddH2O}$, $2 \mu \mathrm{L} \mathrm{cDNA}$, and $1 \mu \mathrm{L}$ with $10 \mu \mathrm{M}$ of each of the forward and reverse primer (Table 1). Amplification conditions were as follows: 2-min preincubation at $50^{\circ} ; 10$-min at $95^{\circ} \mathrm{C}$ for enzyme activation; and 40 cycles at $95^{\circ} \mathrm{C}$ denaturation for $10 \mathrm{~s}, 550 \mathrm{C}$ annealing for $30 \mathrm{~s}$ and $720 \mathrm{C}$ extension for $30 \mathrm{~s}$. Gene expression was normalized with $18 \mathrm{~s}$ mRNA levels. The comparative threshold cycle (Ct) method, that is, the 2- $\Delta \Delta \mathrm{Ct}$ method was used to calculate fold amplification. [26] 
Table 1

Primers for real-time qPCR used in this study

\begin{tabular}{|c|c|c|}
\hline Gene & Primer & Sequence $5^{\prime}$ to $3^{\prime}$ \\
\hline Type II Collagen & Forward & AAG-GGA-CAC-CGA-GGT-TTC-ACT-GG \\
\hline & Reverse & GGG-CCT-GTT-TCT-CCT-GAG-CGT \\
\hline Aggrecan & Forward & CAG-TGC-GAT-GCA-GGC-TGG-CT \\
\hline & Reverse & CCT-CCG-GCA-CTC-GTT-GGC-TG \\
\hline Runx2 & Forward & CCG-CAC-GAC-AAC-CGC-ACC-AT \\
\hline & Reverse & CGC-TCC-GGC-CCA-CAA-ATC-TC \\
\hline MMP-3 & Forward & TTG-TCC-TTC-GAT-GCA-GTC-AG \\
\hline & Reverse & AGA-CGG-CCA-AAA-TGA-AGA-GA \\
\hline MMP-13 & Forward & GGA-CCT-TCT-GGT-CTT-CTG-GC \\
\hline & Reverse & GGA-TGC-TTA-GGG-TTG-GGG-TC \\
\hline Type X Collagen & Forward & CCA-GGT-GTC-CCA-GGA-TTC-CC \\
\hline & Reverse & CAA-GCG-GCA-TCC-CAG-AAA-GC \\
\hline 18S RNA & Forward & CGG-CTA-CCA-CAT-CCA-AGG-AA \\
\hline & Reverse & GCT-GGA-ATT-ACC-GCG-GCT \\
\hline
\end{tabular}

Synovial fluid lavages were collected from the knees as soon as euthanasia. [23] The knee was shaved and prepped with betadine. $50 \mu \mathrm{L}$ of isotonic saline was injected into right knees of each animal through the inferior patellar tendon. The joint capsule was visibly distended. The knee was then manually cycled through flexion and extension 10 times to distribute the fluid within the joint before collection via joint aspiration. Another twice procedures were repeated. This technique typically yielded $80-100 \mu \mathrm{l}$ of fluid from each animal. Then the synovial fluid was centrifuged at 2,000 $\mathrm{g}$ for 10 minutes to remove cells and debris and then was frozen at $-80^{\circ} \mathrm{C}$ until analysis. Once thawed, MMP-13 and sulfatedglycosaminoglycans (sGAG) were measured in the synovial fluid lavages. MMP-13 content was measured in the SF samples using ELISA according to the instructions of the manufacturer (Uscn Life Science). Colorimetric density on the developed plates was determined using a microplate reader (Model BF10000; Packard) set to $450 \mathrm{~nm}$. ELISA analysis of each sample was performed in duplicate. For evaluation of matrix PG release, we used the metachromatic dye 1, 9-dimethylmethylene blue (DMMB) (SigmaAldrich, Gillingham, UK) assay to quantify the amount of sGAG in the SF samples according to manufacturer's instruction. The concentrations of sGAG were obtained from a spectrophotometric reader at $540 \mathrm{~nm}$ (Thermo Scientific Microplate Reader, UK). 


\section{Statistical analysis}

Statistical analyses were performed using SPSS 13.0 software (SPSS Inc., Chicago, IL, USA). One-way ANOVA were used to compare the concentrations of MMP-13 and levels of mRNA in different groups. All data are expressed as mean \pm SD. $p$ values less than 0.05 were considered statistically significant.

\section{Results}

Gross morphological cartilage lesion and fibrillation in the rat tibia plateau were visualized by Indian ink staining. We found that intra-articular HA could not attenuate posttraumatic OA macroscopically compared to $\mathrm{A} 2 \mathrm{M}$ treatment. Comparing with the HA-treated group, there was a significant decrease in the OA score in A2M-treated rats as compared with rats that underwent ACLT and HA/saline treatment. After treated by A2M, decreased India ink staining and a smoother surface were observed compared to that in the HA-treated groups (Fig. 1A). The Indian ink staining Meacham grading system indicated that A2M-treated group had less cartilage lesion and fibrillation than HA-treated or saline-treated group $(p=$ $0.001)$, but more than in the sham control group $(p=0.008)$ (Fig. 1B). And there is no significant difference between HA-treated group and saline-treated group $(p=0.378)$.

Then we value the effect of various treatments by the Histological staining. It is obviously that the supplemental intra-articular A2M has the advantage over HA in attenuation of posttraumatic OA pathogenesis in a rat model of ACLT. Firstly, we found a significant decrease in the OA score in A2Mtreated rats as compared with rats that underwent ACLT and saline/HA treatment. After treatment with $\mathrm{A} 2 \mathrm{M}$, stronger Safranin $\mathrm{O}$ staining, more intact surface and more cellularity but less chondrocyte cloning, and less fibrillation were observed than in the saline/HA-treated groups, but weaker staining than that from control rats underwent sham operation (Fig. 2A). Cartilage from rats administered the injection of HA had almost similar staining and surface compared with cartilage from rats without treatment (Saline group) (Fig. 2A). OARSI histologic grading system scores in A2M-treated group suggested mild degeneration (mean $\pm S D, 9.82 \pm 4.0$ ), while cartilage damage in saline-treated group and HA-treated group were significantly more severe $(18.2 \pm 3.2$ in the ACLT + saline group and $16.1 \pm 3.8$ in the ACLT + HA group; $P<0.01)$. It should be noted that there was no significant statistical difference between ACLT + saline group and ACLT + HA group $(P=0.062)$. The cartilage from rats that underwent sham operation had the least amount of damage $(0.43 \pm 0.4 ; P<0.01)$ (Fig. 2B).

Next, Immunohistochemistry was performed to determine the expression of type II collagen and MMP-13. Type II collagen content was higher in cartilage in the ACLT + A2M and Sham + Saline groups than in the ACLT + HA group and ACLT + Saline group (Fig. 3A). In contrast, MMP-13 was elevated in cartilage of the HA-treated group compared to A2M-treated cartilage, in response to this, the group with higher MMP-13 level suffered critical cartilage injury. (Figs. 3B).

Additionally, Cartilage damage was associated with a change in the levels of MMP-13 in joint lavage fluid. The mean \pm SD MMP-13 level of rats that underwent ACLT and A2M treatment was $0.63 \pm$ 
$0.05 \mu \mathrm{g} / \mathrm{ml}$, which was higher than that in sham operation group $(0.266 \pm 0.03 \mu \mathrm{g} / \mathrm{ml} ; \mathrm{P}=1.85 \mathrm{E}-05)$, while much lower than that in rats administered injection of HA $(1.168 \pm 0.18 \mu \mathrm{g} / \mathrm{ml} ; \mathrm{P}=4.65 \mathrm{E}-05)$ and rats administered injection of saline $(1.34 \pm 0.19 \mu \mathrm{g} / \mathrm{ml}, P=1.27 \mathrm{E}-06)$. However, there was no statistical difference between ACLT + HA group and ACLT + Saline group (Fig. 4).

Besides, we examined the sulphated glycosaminoglycan (sGAG) levels in synovial fluid groups with different treatment strategies (Fig. 5). sGAG is an integral component part in cartilage matrix. However, aGAG detected in synovial fluid indicates the destruction of the articular cartilage. The sGAG level in A2Mtreated group was $331.68 \pm 75.47 \mu \mathrm{g} / \mathrm{ml}$, which was higher than the sham group $(213.74 \pm 44.14 \mu \mathrm{g} / \mathrm{ml}$; $P=0.008)$. But it is noteworthy that the synovial fluid sGAG level of the A2M group was significant lower than no-treatment group $(567.70 \pm 75.23 \mu \mathrm{g} / \mathrm{ml} ; \mathrm{P}=0.001)$ (Fig. 5). This result demonstrated the protective effect of supplemental intra-articular A2M injection. And we found ACLT operated rats in group with saline or HA possessed higher quantities of synovial fluid SGAG $(576.56 \pm 44.14 \mu \mathrm{g} / \mathrm{ml})$ than those treated by A2M ( $P=9.33 \mathrm{E}-05)$, also much higher than rats in the control group too ( $P=2.77 \mathrm{E}-07)$ (Fig. 5$)$. Meanwhile there was no statistical difference between ACLT + saline group and ACLT + HA group $(P=$ 0.825) (Fig. 5).

Also, we performed qPCR to investigate the expression of related gene. Real-time qPCR results indicated that the expression of mRNA for type II collagen and aggrecan increased in the group with supplemental intra-articular A2M, while the levels of mRNA for Runx2, MMP-3, MMP-13, and type X collagen went down (Fig. 6). By looking at HA treated group only, the levels of aggrecan and type II collagen mRNA were obviously low, in contrary, the level of mRNA for Runx2, MMP-3, MMP-13, and type X collagen were over expressed (Fig. 6). Type II collagen mRNA level in rats that underwent ACLT and A2M treatment was significantly higher than that in rats underwent ACLT with HA or saline injection, and there was no significant difference between the latter two groups. In the groups underwent sham operation and treated by $A 2 M$, the aggrecan mRNA levels were significantly higher than the groups with saline and HA injection. In contrast, levels of mRNA for Runx2, MMP-3, MMP-13, and type X collagen in rats that underwent ACLT and saline/HA treatment were much higher than other two groups. (Fig. 6).

\section{Discussion}

Post-trauma Osteoarthritis is a sort of long course disease initiated by joint injury and consequent intraarticular inflammation, and its process is irreversible $[27,28]$. Thus, to delay the cartilaginous degeneration is the main purpose of non-surgical treatment for osteoarthritis. Chronic inflammation is a critical risk factor of the cartilage destruction during the process of osteoarthritis, therefore, it is believed that the reduction of the intra-articular inflammation will retard the degeneration of cartilage [28]. Accordingly, we assumed that supplemental intra-articular injection of $A 2 \mathrm{M}$, a autogeneic broad spectrum proteinase inhibitor, could ameliorate cartilage destruction induced by inflammation.

We set four groups to compare the protective effect of $A 2 \mathrm{M}$ and $\mathrm{HA}$, two experimental groups received ACLT were treated with A2M and HA injection separately. Results of India ink staining and Safranin- 
O/fast green staining have been consistent. All groups received ACLT showed articular cartilage defects and outcropping of subchondral bone, which all were typical pathological changes of osteoarthritis [29]. However, there was little normal cartilage remaining in HA treated group, indicate the cartilage damages in HA group and non-treated group had no significant difference, hence the protective effect of HA is weak. This phenomenon echoed the results of clinical trials and meta-analyses, proving the uncertainty of curative effect of HA [30-32].

In A2M treated group, cartilage damage occurred similarly but obviously slighter than the HA group and non-treatment group. It could be observed that $\mathrm{A} 2 \mathrm{M}$-treated group had relatively intact surface and more chondrocyte cloning than non-treatment group and HA-treated group. Although the cartilage was inevitable after supplemental A2M injection, the protective effect of A2M was still very evident. Worthy of note was that OARSI histologic grading system scores in A2M-treated group also showed significant decrease, suggesting mild degeneration.

The major pathological changes of PTOA is the chronic inflammation secondary to joint injury. Due to the increasingly producing of pro-inflammatory cytokine and matrix metalloproteinase, the inflammatory damage of the articular tissue outrun regulation. Thus, relatively forceful way to analyze and compare the protective effects between $\mathrm{HA}$ and $\mathrm{A} 2 \mathrm{M}$ is to study whether the intervention can inhibit the destructive cytokine and enzyme.

Accordingly, we examined more biological indicator of cartilage degeneration. Immunohistochemistry was carried out to test distribution of types II collagen and MMP-13 in cartilage. Type II collagen is produced by chondrocyte, and the level of type II collagen in degenerated cartilage with lower chondrocyte cloning will reduce [33]. While MMP-13 is a kind of enzyme catalyzing destruction of cartilage. MMP-13 induces the degradation of proteoglycan and type II collagen; thus it is used as an important indicator of cartilage damage. [34-36] in A2M injection group we detected higher type II collagen level and lower MMP-13 level, hence the protective effect of supplemental A2M injection could be confirmed. Besides we examined the sGAG concentration in synovial fluid from each group. Comparing to the non-treatment group, the synovial fluid sGAG level of the A2M group was lower. Quantitatively assessing sGAG level in synovial fluid could reflect the destruction of the cartilage extracellular matrix [37]. It is probably that the protective effect of A2M lead to lower sGAG in the group treated by this novel therapy.

In qPCR result, it is obviously that the supplemental A2M injection reduced the levels of mRNA for Runx2, MMP-3, MMP-13, and type X collagen. However, levels of mRNA for all that above in rats that underwent ACLT and saline/HA treatment were much higher than other two groups. These data suggested that A2M can protect cartilage in vivo by decreasing gene expression of catabolic factors and hypertrophic markers, as well as by increasing anabolic gene expression.

It is also apparently that the HA failed to achieved desired therapeutic effect. Immunohistochemistry Staining showed that the cartilage degeneration in rat knees with HA injection were still severe, meanwhile, the MMP-13 level was extremely higher than the sham operation group and A2M group. 
According to the quantitative assay of MMP13 level in synovial fluid, no significant disparity was found between HA injection group and non-treatment group. Similarly, there was no diversity between the sGAG level of HA group and non-treatment group. The result of qPCR also indicated that the HA treatment failed to prevent cartilage destruction.

As a lot of research, HA cannot display the protective effect to the injured joint tissue. A commonly accepted view is that the intra articular injection of HA can work as visco-supplementation [19]. But as early as the 1980s, a study suggested that injecting HA could not raise the specific viscosities of synovial fluid in experimental damaged joint [38]. One of the important reasons is the high molecular weight $\mathrm{HA}(\mathrm{HMW}-\mathrm{HA})$ will be hydrolyzed to low molecular weight $\mathrm{HA}(\mathrm{LMW}-\mathrm{HA})$ in the wound healing process, and LMW-HA cannot increase the specific viscosities of synovial fluid [39]. Furthermore, many studies believe that the LWM-HA is an active pro-inflammatory stimulator, even inducing metalloproteinases production [40-42].

Besides, there is a sort of proteoglycans called aggrecan on articular cartilage which binds to HA, providing elasticity of cartilage [43]. When PTOA happens, aggrecan on joint cartilage is dissociated, leading to loss of HA and erosion of cartilage, and finally cartilage degeneration [44]. And there is no evidence that supplement of HA without aggrecan will contribute to cartilage repairment. Additionally, previous study suggest that the HA have no influence on proteoglycan concentration in PTOA [45]. And our research also came to conclusion that there was no significant difference on mRNA level of aggrecan between the HA treated group and non-treatment group.

Although, intra-articular injection of HA supplies HMW-HA, but the change is temporary. Through the HA injection therapy, synovial fluid is difficult to recover to normal standard. While any change in molecular weight and concentration of HA in synovial fluid will aggravate the articular cartilage damage. For the reasons above, the HA treatment has been no longer recommended by American Academy of Orthopaedic Surgeons (AAOS) [22].

The reason we've decided to used $\mathrm{A} 2 \mathrm{M}$ as an alternative therapy to replace uncertain HA therapy. Not only because $\mathrm{A} 2 \mathrm{M}$ is able to protect articular cartilage against chronic inflammation, but also A2M has high biosafety and less side effects.

There are many studies working on solve the inflammation caused cartilage destruction in osteoarthritis. In this direction, existing and examining therapies are intra-articular calcium channel blocking anesthetics, corticosteroid injection, mesenchymal stem/stromal cells, and platelet-rich plasma [46, 47]. However, some of treatments mentioned above cannot slow down the cartilage degeneration; some are not suitable for prolonged use in clinics. A2M, as a novel therapeutic drug for knee OA, has a pretty good prospect in solving the shortcomings mentioned above. Compared with other drugs above, A2M is an autologous proteinase inhibitor so that it has no autoimmune rejection. Additionally, A2M injection contains only one kind of active ingredient, but inhibits various inflammatory factors and degenerative proteinase, due to broad-spectrum anti-inflammation effect of A2M. Therefore, A2M injection eliminates above-mentioned limitations. As a result, the safety is guaranteed and risk is controllable. 
New developed technologies allowed us to concentrate A2M molecule from autologous plasma of patients, then inject $\mathrm{A} 2 \mathrm{M}$-rich preparation into joint to compensate for the low level of A2M in synovial fluid. [48] Furthermore, the variants of A2M called CYT-108 can be synthesized according to A2M's molecular properties. Doubtless synthetic A2M is more economic and convenient, and the inhibitory effect of CYT-108 A2M far exceeded wild-type A2M [49].

\section{Conclusion}

This paper proposed to examine the uncertainty of HA treatment in animal models. And test the articularly protective effect in osteoarthritis of $\mathrm{A} 2 \mathrm{M}$ in the meantime. The results demonstrated that the $\mathrm{A} 2 \mathrm{M}$ can postpone retrograde degeneration of joint cartilage effectively. And, as expected, HA treatment did not conduct protective effect obviously. These observations indicate that A2M is a promising antiinflammation treatment for osteoarthritis, and it can be used as the replacement for HA.

\section{Abbreviations}

PTOA: Post-Traumatic Osteoarthritis

HA: Hyaluronic Acid

ACLT: Anterior Cruciate Ligament Transection

A2M : Alpha-2-macroglobulin

SF: Synovial Fluid

MMPS: Matrix Metalloproteinases

MCP: Monocyte chemoattractant protein

sGAG: sulfated-Glycosaminoglycans

DMMB: Dimethylmethylene Blue

HMW-HA: High Molecular Weight HA

LMW-HA: Low Molecular Weight HA

\section{Declarations}

\section{Availability of data and materials}

All data generated and analyzed in this study are disclosed in this article. 


\section{Funding}

This project was supported by the National Natural Science Foundation of China (Grant No. 81572207, 81201435), and Cultivate Scientific Research Excellence Programs of Higher Education Institutions in Shanxi (CSREP), China.

\section{Authors' contributions}

MZ participated in the study design, wrote the manuscript. YG, RY and ZW performed most of the experiments. BZ and $Y C$ analyzed data and participated in the interpretation of the data and the revision of the manuscript. SW and LW conceived the in vivo study and participated in its design and data analysis and revised the manuscript. All authors have read and approved the final manuscript.

\section{Conflict of interest}

The authors declare that they have no conflict of interest.

\section{Ethics approval and consent to participate}

This study did not need consent from any individuals/patients because no humans were involved in this study. Ethical approval was needed for this study and it was approved by the Institutional Review Board and the Institutional Animal Care and Use Committee of the Shanxi Medical University (approved protocol 2018LL039)

\section{Disclosures}

We have nothing to disclose.

\section{References}

1. Minguzzi M, Cetrullo S, D'Adamo S, Silvestri Y, Flamigni F, Borzi RM: Emerging Players at the Intersection of Chondrocyte Loss of Maturational Arrest, Oxidative Stress, Senescence and LowGrade Inflammation in Osteoarthritis. Oxid Med Cell Longev 2018, 2018:3075293.

2. Pap T, Korb-Pap A: Cartilage damage in osteoarthritis and rheumatoid arthritis-two unequal siblings. Nat Rev Rheumatol 2015, 11(10):606-615.

3. Heinegard D, Saxne T: The role of the cartilage matrix in osteoarthritis. Nat Rev Rheumato/2011, $7(1): 50-56$. 
4. Gelber AC, Hochberg MC, Mead LA, Wang NY, Wigley FM, Klag MJ: Joint injury in young adults and risk for subsequent knee and hip osteoarthritis. Ann Intern Med 2000, 133(5):321-328.

5. Sward P, Struglics A, Englund M, Roos HP, Frobell RB: Soft tissue knee injury with concomitant osteochondral fracture is associated with higher degree of acute joint inflammation. Am J Sports Med 2014, 42(5):1096-1102.

6. Elsaid KA, Fleming BC, Oksendahl HL, Machan JT, Fadale PD, Hulstyn MJ, Shalvoy R, Jay GD: Decreased lubricin concentrations and markers of joint inflammation in the synovial fluid of patients with anterior cruciate ligament injury. Arthritis Rheum 2008, 58(6):1707-1715.

7. Cameron ML, Fu FH, Paessler HH, Schneider M, Evans $\mathrm{CH}$ : Synovial fluid cytokine concentrations as possible prognostic indicators in the ACL-deficient knee. Knee Surg Sports Traumatol Arthrosc 1994, 2(1):38-44.

8. Tang Z, Yang L, Xue R, Zhang J, Wang Y, Chen PC, Sung KL: Differential expression of matrix metalloproteinases and tissue inhibitors of metalloproteinases in anterior cruciate ligament and medial collateral ligament fibroblasts after a mechanical injury: involvement of the p65 subunit of NF-kappaB. Wound Repair Regen 2009, 17(5):709-716.

9. Attia E, Brown H, Henshaw R, George S, Hannafin JA: Patterns of gene expression in a rabbit partial anterior cruciate ligament transection model: the potential role of mechanical forces. Am J Sports Med 2010, 38(2):348-356.

10. Aini H, Ochi H, Iwata M, Okawa A, Koga D, Okazaki M, Sano A, Asou Y: Procyanidin B3 prevents articular cartilage degeneration and heterotopic cartilage formation in a mouse surgical osteoarthritis model. PLoS One 2012, 7(5):e37728.

11. Haslauer CM, Elsaid KA, Fleming BC, Proffen BL, Johnson VM, Murray MM: Loss of extracellular matrix from articular cartilage is mediated by the synovium and ligament after anterior cruciate ligament injury. Osteoarthritis Cartilage 2013, 21(12):1950-1957.

12. Nagai T, Sato M, Kobayashi M, Yokoyama M, Tani Y, Mochida J: Bevacizumab, an anti-vascular endothelial growth factor antibody, inhibits osteoarthritis. Arthritis Res Ther 2014, 16(5):427.

13. Cuellar JM, Scuderi GJ, Cuellar VG, Golish SR, Yeomans DC: Diagnostic utility of cytokine biomarkers in the evaluation of acute knee pain. J Bone Joint Surg Am 2009, 91(10):2313-2320.

14. Cuellar VG, Cuellar JM, Golish SR, Yeomans DC, Scuderi GJ: Cytokine profiling in acute anterior cruciate ligament injury. Arthroscopy 2010, 26(10):1296-1301.

15. Lieberthal J, Sambamurthy N, Scanzello CR: Inflammation in joint injury and post-traumatic osteoarthritis. Osteoarthritis Cartilage 2015, 23(11):1825-1834.

16. Sottrup-Jensen L: Alpha-macroglobulins: structure, shape, and mechanism of proteinase complex formation. J Biol Chem 1989, 264(20):11539-11542.

17. Wang S, Wei X, Zhou J, Zhang J, Li K, Chen Q, Terek R, Fleming BC, Goldring MB, Ehrlich MG et al: Identification of a2-Macroglobulin as a Master Inhibitor of Cartilage-Degrading Factors That Attenuates the Progression of Posttraumatic Osteoarthritis. Arthritis \& Rheumatology 2014, 66(7):1843-1853. 
18. Demirag B, Sarisozen B, Durak K, Bilgen OF, Ozturk C: The effect of alpha-2 macroglobulin on the healing of ruptured anterior cruciate ligament in rabbits. Connect Tissue Res 2004, 45(1):23-27.

19. Altman RD, Manjoo A, Fierlinger A, Niazi F, Nicholls M: The mechanism of action for hyaluronic acid treatment in the osteoarthritic knee: a systematic review. BMC Musculoskelet Disord 2015, 16:321.

20. Lo GH, LaValley M, McAlindon T, Felson DT: Intra-articular hyaluronic acid in treatment of knee osteoarthritis: a meta-analysis. JAMA 2003, 290(23):3115-3121.

21. Arrich J, Piribauer F, Mad P, Schmid D, Klaushofer K, Mullner M: Intra-articular hyaluronic acid for the treatment of osteoarthritis of the knee: systematic review and meta-analysis. CMAJ 2005, 172(8):1039-1043.

22. Cook R, Davidson P, White A, Centre ND: Partial knee replacement could be first choice for some patients with osteoarthritis. BMJ 2019, 367:15994.

23. Wei L, Fleming BC, Sun X, Teeple E, Wu W, Jay GD, Elsaid KA, Luo J, Machan JT, Chen Q: Comparison of differential biomarkers of osteoarthritis with and without posttraumatic injury in the Hartley guinea pig model. J Orthop Res 2010, 28(7):900-906.

24. Meachim G: Light microscopy of Indian ink preparations of fibrillated cartilage. Ann Rheum Dis 1972, 31(6):457-464.

25. Pritzker KP, Gay S, Jimenez SA, Ostergaard K, Pelletier JP, Revell PA, Salter D, van den Berg WB: Osteoarthritis cartilage histopathology: grading and staging. Osteoarthritis Cartilage 2006, 14(1):1329.

26. Wang S, Wei X, Zhou J, Zhang J, Li K, Chen Q, Terek R, Fleming BC, Goldring MB, Ehrlich MG et al: Identification of alpha2-macroglobulin as a master inhibitor of cartilage-degrading factors that attenuates the progression of posttraumatic osteoarthritis. Arthritis Rheumatol 2014, 66(7):18431853.

27. Yamada EF, Bobinski F, Martins DF, Palandi J, Folmer V, da Silva MD: Photobiomodulation therapy in knee osteoarthritis reduces oxidative stress and inflammatory cytokines in rats. J Biophotonics 2020, 13(1):e201900204.

28. Irie K, Uchiyama E, Iwaso H: Intraarticular inflammatory cytokines in acute anterior cruciate ligament injured knee. Knee 2003, 10(1):93-96.

29. Takeda H, Nakagawa T, Nakamura K, Engebretsen L: Prevention and management of knee osteoarthritis and knee cartilage injury in sports. Br J Sports Med 2011, 45(4):304-309.

30. Bannuru RR, McAlindon TE, Sullivan MC, Wong JB, Kent DM, Schmid CH: Effectiveness and Implications of Alternative Placebo Treatments: A Systematic Review and Network Meta-analysis of Osteoarthritis Trials. Ann Intern Med 2015, 163(5):365-372.

31. Bannuru RR, Schmid CH, Kent DM, Vaysbrot EE, Wong JB, McAlindon TE: Comparative effectiveness of pharmacologic interventions for knee osteoarthritis: a systematic review and network metaanalysis. Ann Intern Med 2015, 162(1):46-54.

32. Niemela TM, Tulamo RM, Hielm-Bjorkman AK: A randomised, double-blinded, placebo-controlled clinical study on intra-articular hyaluronan treatment in equine lameness originating from the 
metacarpophalangeal joint. BMC Vet Res 2016, 12:60.

33. Poole AR, Kobayashi M, Yasuda T, Laverty S, Mwale F, Kojima T, Sakai T, Wahl C, El-Maadawy S, Webb $\mathrm{G}$ et al: Type II collagen degradation and its regulation in articular cartilage in osteoarthritis. Ann Rheum Dis 2002, 61 Suppl 2:ii78-81.

34. Gu R, Shi Y, Huang W, Lao C, Zou Z, Pan S, Huang Z: Theobromine mitigates IL-1beta-induced oxidative stress, inflammatory response, and degradation of type II collagen in human chondrocytes. Int Immunopharmacol 2020, 82:106226.

35. Alamgeer, Hasan UH, Uttra AM, Qasim S, Ikram J, Saleem M, Niazi ZR: Phytochemicals targeting matrix metalloproteinases regulating tissue degradation in inflammation and rheumatoid arthritis. Phytomedicine 2020, 66:153134.

36. Burrage PS, Mix KS, Brinckerhoff CE: Matrix metalloproteinases: role in arthritis. Front Biosci 2006, 11:529-543.

37. Jin Y, Chen X, Gao ZY, Liu K, Hou Y, Zheng J: The role of miR-320a and IL-1beta in human chondrocyte degradation. Bone Joint Res 2017, 6(4):196-203.

38. Hilbert BJ, Rowley G, Antonas KN, McGill CA, Reynoldson JA, Hawkins CD: Changes in the synovia after the intra-articular injection of sodium hyaluronate into normal horse joints and after arthrotomy and experimental cartilage damage. Aust Vet J 1985, 62(6):182-184.

39. Aviad AD, Houpt JB: The molecular weight of therapeutic hyaluronan (sodium hyaluronate): how significant is it? J Rheumatol 1994, 21(2):297-301.

40. Chen WY, Abatangelo G: Functions of hyaluronan in wound repair. Wound Repair Regen 1999, 7(2):79-89.

41. Stern R, Asari AA, Sugahara KN: Hyaluronan fragments: an information-rich system. Eur J Cell Biol 2006, 85(8):699-715.

42. Jiang D, Liang J, Noble PW: Hyaluronan in tissue injury and repair. Annu Rev Cell Dev Biol 2007, 23:435-461.

43. Tortorella MD, Malfait AM, Deccico C, Arner E: The role of ADAM-TS4 (aggrecanase-1) and ADAMTS5 (aggrecanase-2) in a model of cartilage degradation. Osteoarthritis Cartilage 2001, 9(6):539552.

44. Tortorella MD, Burn TC, Pratta MA, Abbaszade I, Hollis JM, Liu R, Rosenfeld SA, Copeland RA, Decicco CP, Wynn R et al: Purification and cloning of aggrecanase-1: a member of the ADAMTS family of proteins. Science 1999, 284(5420):1664-1666.

45. Brandt KD, Smith GN, Jr., Simon LS: Intraarticular injection of hyaluronan as treatment for knee osteoarthritis: what is the evidence? Arthritis Rheum 2000, 43(6):1192-1203.

46. Xie X, Zhang C, Tuan RS: Biology of platelet-rich plasma and its clinical application in cartilage repair. Arthritis Res Ther 2014, 16(1):204.

47. Richards MM, Maxwell JS, Weng L, Angelos MG, Golzarian J: Intra-articular treatment of knee osteoarthritis: from anti-inflammatories to products of regenerative medicine. Phys Sportsmed 2016, 
44(2):101-108.

48. Cuellar JM, Cuellar VG, Scuderi GJ: alpha2-Macroglobulin: Autologous Protease Inhibition Technology. Phys Med Rehabil Clin N Am 2016, 27(4):909-918.

49. Zhang Y, Wei X, Browning S, Scuderi G, Hanna LS, Wei L: Targeted designed variants of alpha-2macroglobulin (A2M) attenuate cartilage degeneration in a rat model of osteoarthritis induced by anterior cruciate ligament transection. Arthritis Res Ther 2017, 19(1):175.

\section{Figures}

A

Femur
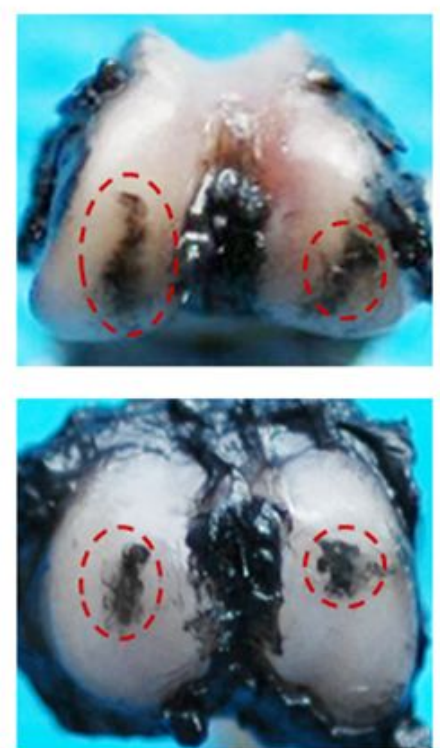

Lat

Med

Tibia
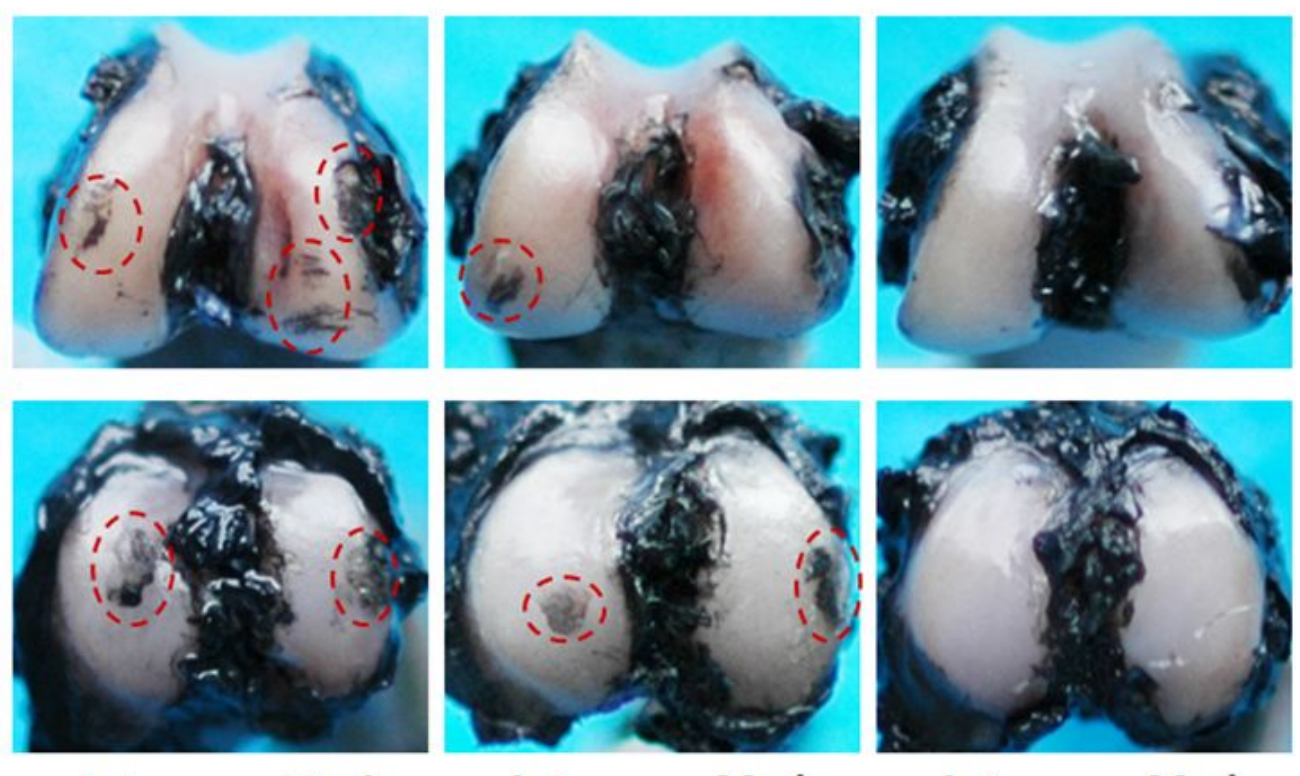

Lat Med

Lat

Med

Lat

Med

B
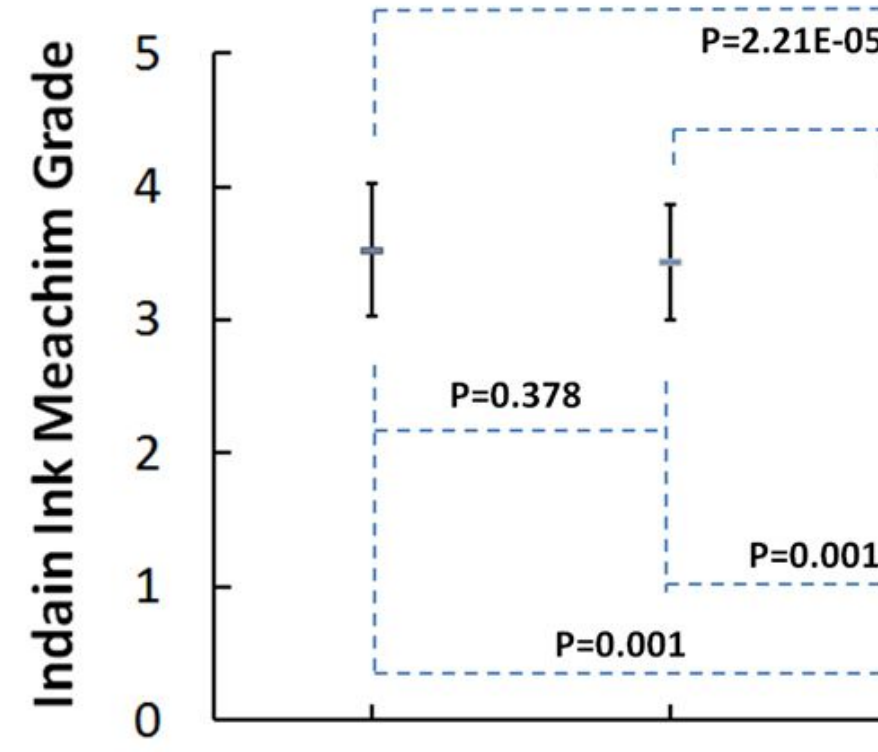

$\mathrm{P}=2.21 \mathrm{E}-05$

$P=1.02 E-05$

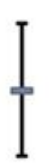

I
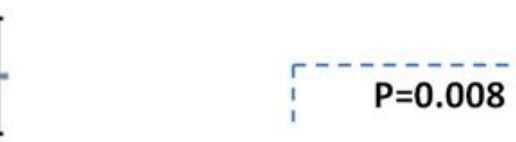

$P=0.378$
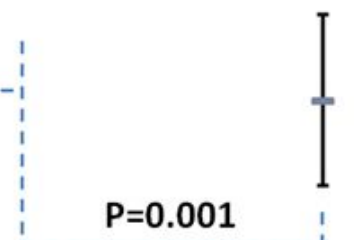

$\mathrm{P}=0.001$

ACLT+Saline 
Figure 1

Intra-articular HA could not attenuate posttraumatic OA macroscopically. (A) Decreased India ink staining and a smoother surface were detected in the articular cartilage of A2M-treated animals as compared to ACLT and saline/HA treatment groups. (B) The Indian ink Meachim Grade score indicated that cartilage damage was much severe in rats that underwent ACLT and saline/HA treatment, while cartilage in rats that underwent sham operation had the least damage. Cartilage damage was also reduced in rats that received $\mathrm{A} 2 \mathrm{M}$ as compared to rats that received the HA treatment. Values are the mean \pm SD.

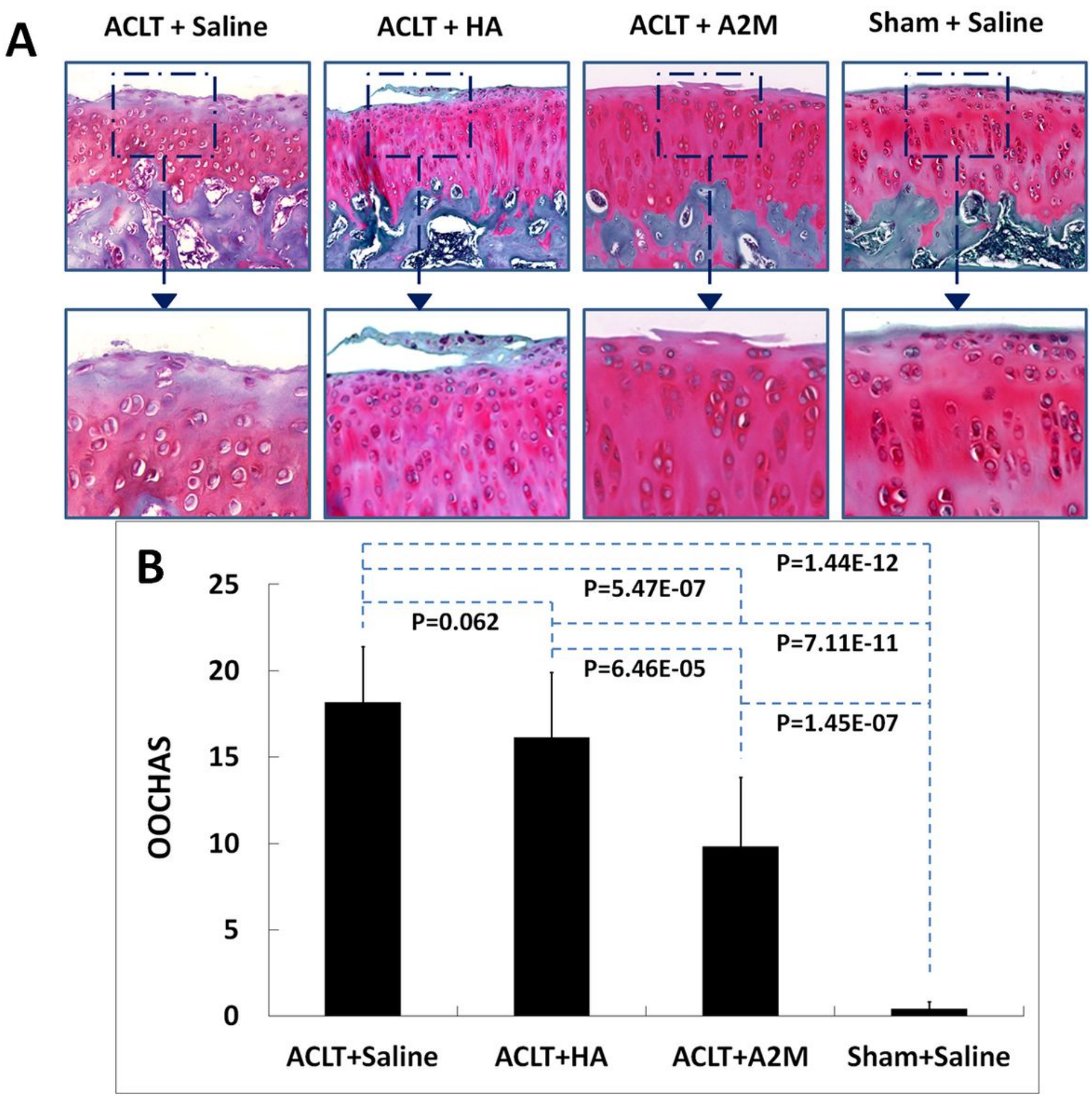

Figure 2 
Posttraumatic OA rats modeled by ACLT suggested that the A2M has more advantage than HA in attenuating the injury. (A) A smoother surface with stronger Safranin $O$ staining was detected in the articular cartilage of A2M-treated animals as compared to HA-treated animals and untreated controls (the bottom panels are higher-magnification views of the boxed areas in the top panels). (B) The Osteoarthritis Research Society International Osteoarthritis Cartilage Histopathology Assessment System (OOCHAS) score indicated that cartilage damage was mild in rats that underwent A2M treatment, while cartilage in rats that underwent sham operation had the least damage. Cartilage damage was aggravated in rats that received the ACLT operation and HA/Saline treatment. And there was no statistical significance between ACLT+saline group and ACLT+HA group. Values are the mean $\pm S D$.

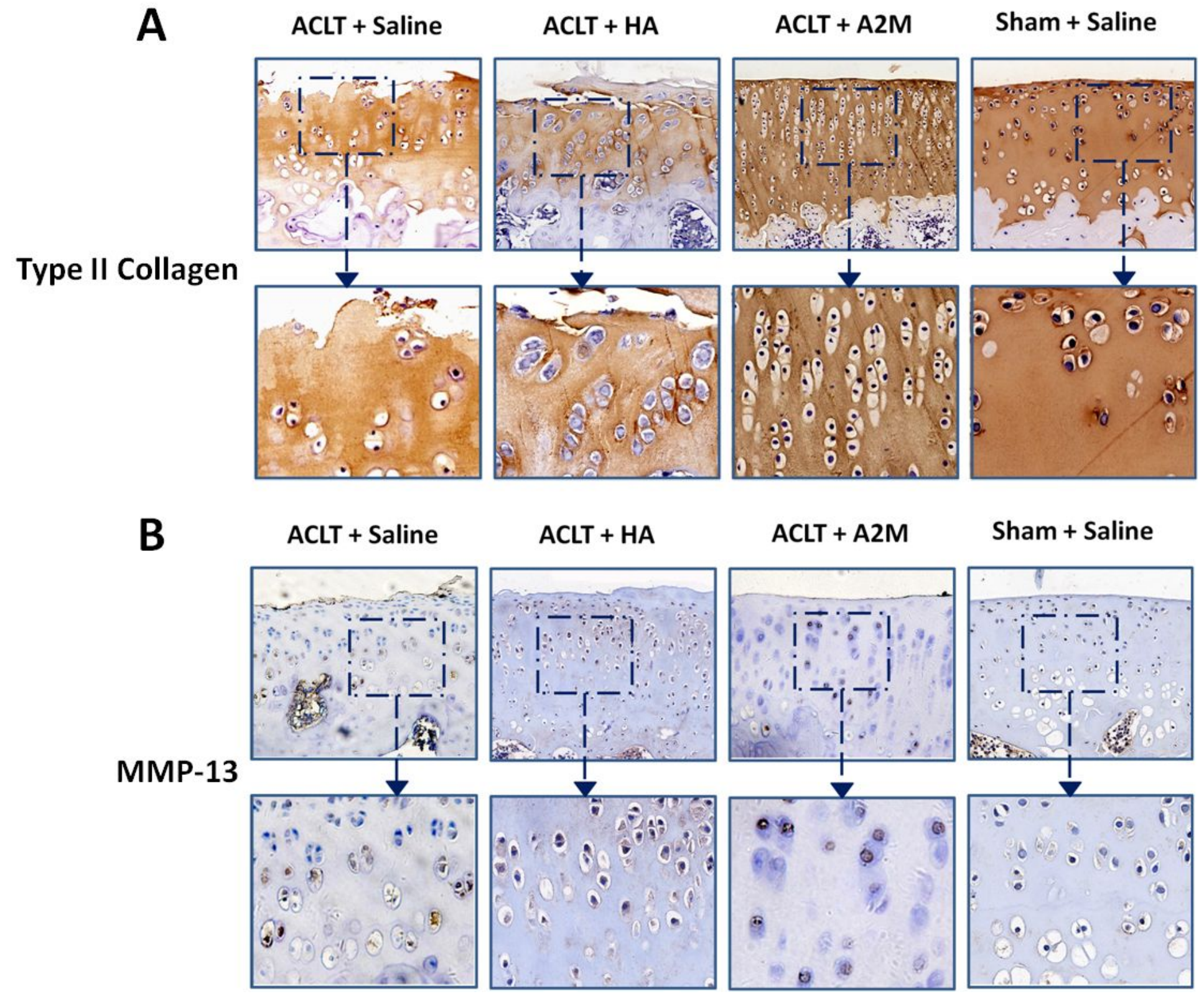

Figure 3

MMP-13 expression was reduced in A2M treated rats, but type II collagen was preserved. (A) Type II collagen expression in articular cartilage was higher in the A2M-treated and the sham-operated rats than 
in rats that underwent ACLT and HA treatment. In contrast, MMP-13 staining was elevated in rats that underwent ACLT and HA treatment, but was lower in the A2M-treated and sham-operated rats, which is consistent with reduced $O A$ damage in these rats. In $A$ and $B$, the bottom panels are higher-magnification views of the boxed areas in the top panels (20x objective lens).

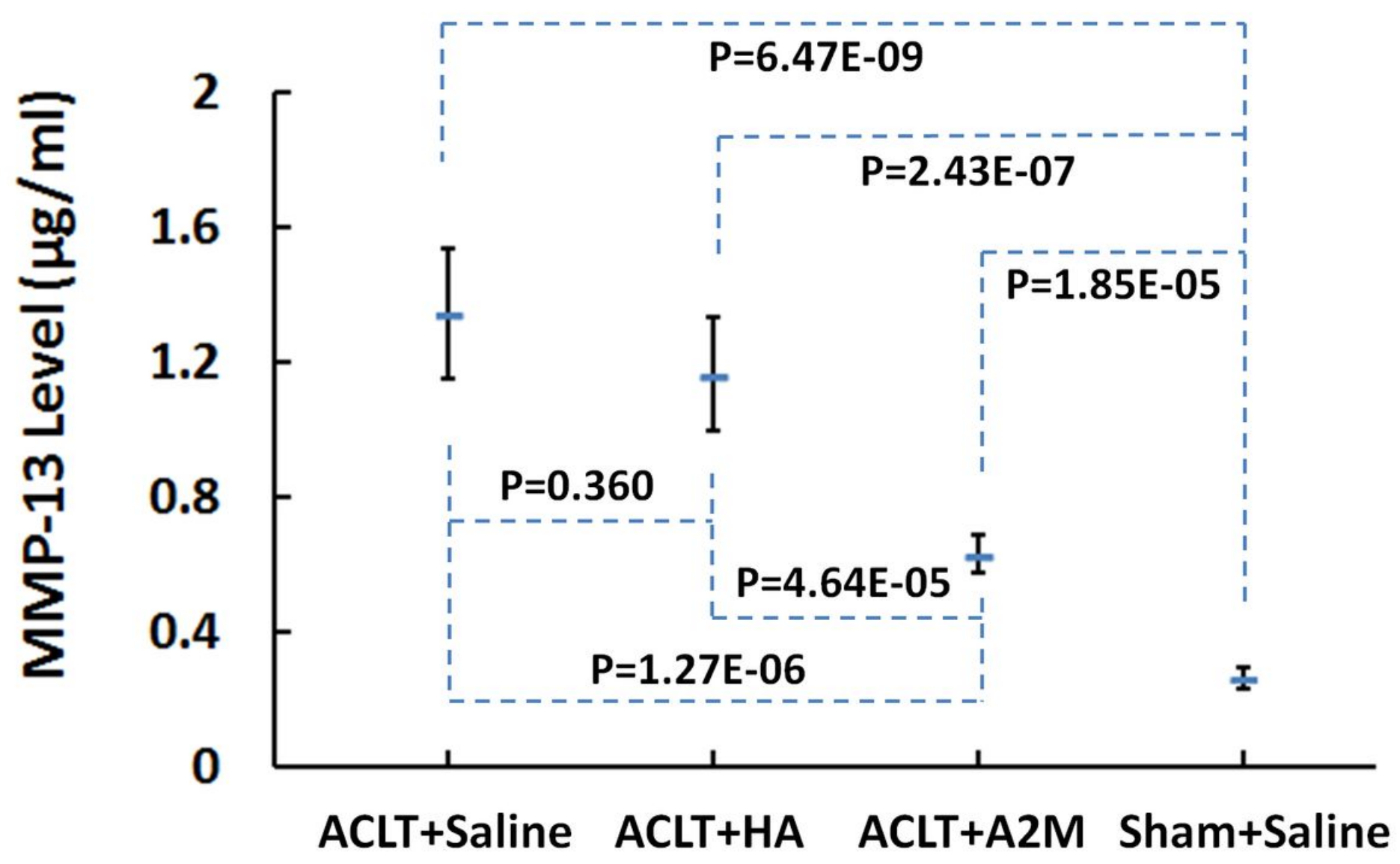

Figure 4

In HA-treated rats, the concentration of MMP-13 in SF was much higher than that in rats that underwent ACLT and A2M treatment and was similar to that in ACLT and saline treated rats. Values are the mean $\pm S D$. 


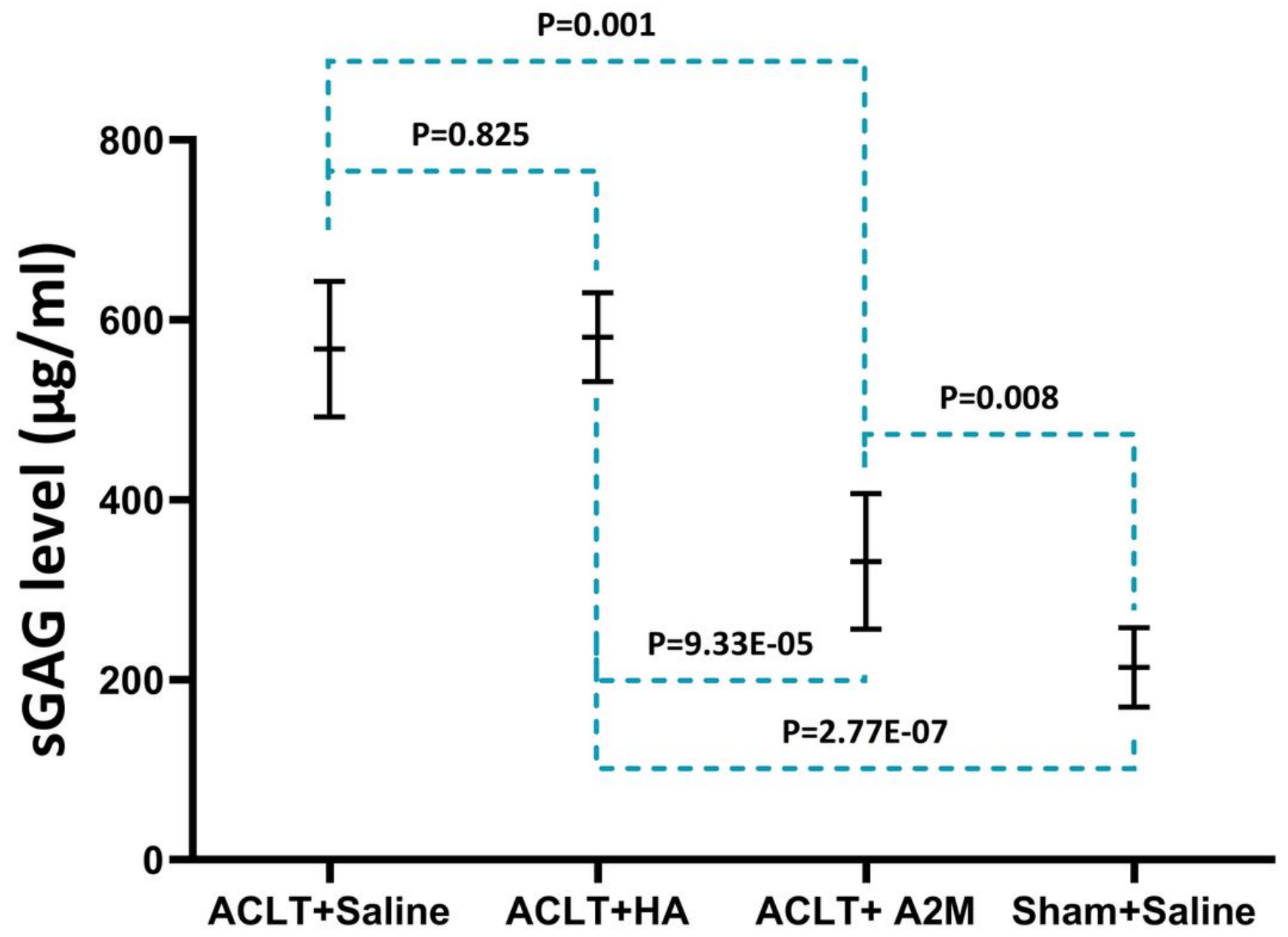

Figure 5

ACLT rats treated by HA had shown more articular cartilage destruction than those treated by $\mathrm{A} 2 \mathrm{M}$. The sGAG concentrations were tested separately in four groups. In HA-treated rats, the concentration of sGAG in SF was much higher than that in rats that underwent ACLT and A2M treatment. No statistical difference was found between ACLT+Saline group and ACLT+ HA group. Values are the mean $\pm S D$. 


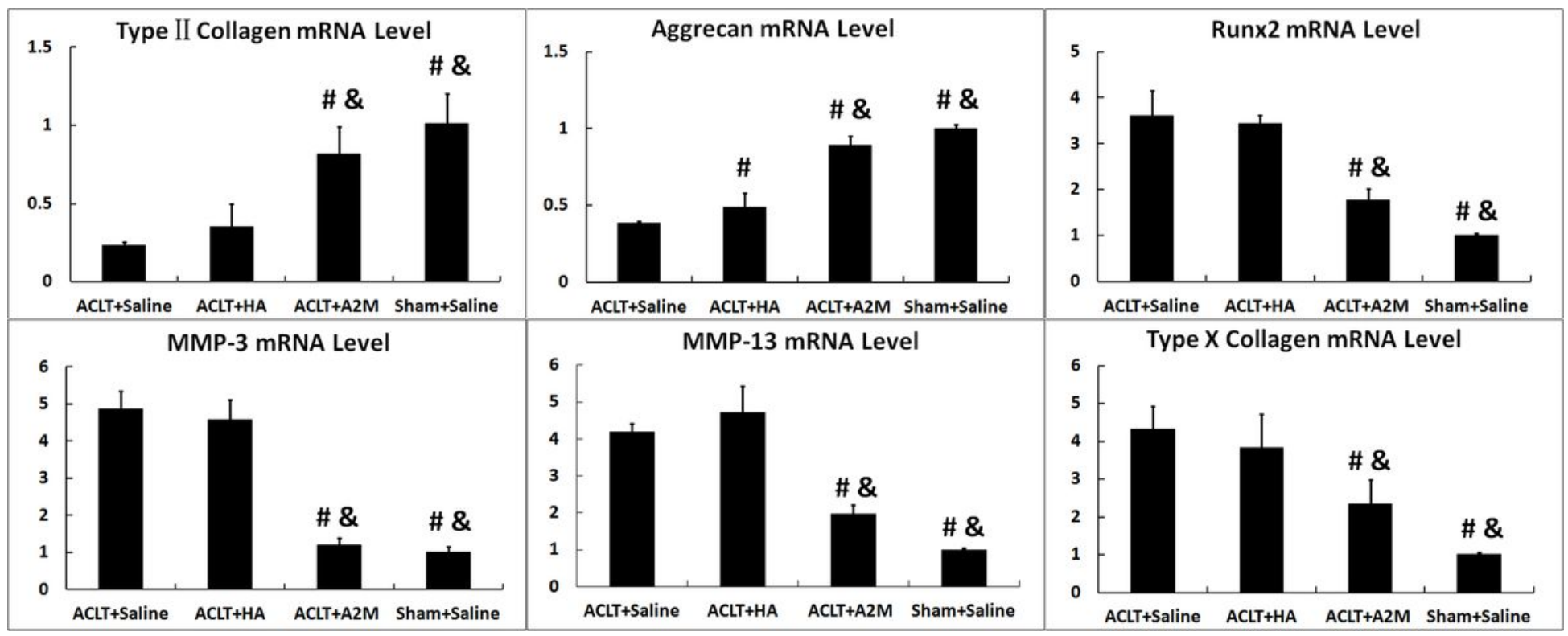

Figure 6

Supplemental intra-articular A2M has the advantage over HA in inhibiting catabolism and enhancing anabolic metabolism in a rat model of ACLT. Levels of mRNA for type II collagen and aggrecan were increased in rats that were administered $\mathrm{A} 2 \mathrm{M}$ as compared to rats that underwent ACLT and saline/HA treatment, suggesting that $\mathrm{A} 2 \mathrm{M}$ has a positive impact on anabolic metabolism. In contrast, Runx2, MMP3, MMP-13, and type $X$ collagen showed the opposite pattern. These genes were expressed at a lower level in rats that were administered A2M as compared to rats that underwent ACLT and saline/HA treatment. Values are the mean \pm SEM. \#: $P<0.01$ versus the ACLT and saline treatment group; \&: $P<0.01$ versus the ACLT and HA treatment group. 